Bull. Korean Math. Soc. 49 (2012), No. 5, pp. 1041-1055

http://dx.doi.org/10.4134/BKMS.2012.49.5.1041

\title{
THE PARAMETER DISTRIBUTION SET FOR A SELF-SIMILAR MEASURE
}

\author{
IN-SOO BAEK
}

\begin{abstract}
The parameter lower (upper) distribution set corresponds to the cylindrical lower or upper local dimension set for a self-similar measure on a self-similar set satisfying the open set condition.
\end{abstract}

\section{Introduction}

A self-similar set is the attractor of an iterated function system (IFS) [8]. Recently, we investigated the relation between spectral classes of a self-similar Cantor set in a set theoretical sense [1]. In this paper, using the parameter distribution, we find the parallel results for the self-similar set (attractor of the IFS consisting of $n(\geq 2)$ similitudes satisfying the OSC (open set condition)) instead of the self-similar Cantor set (attractor of the IFS consisting of 2 similitudes satisfying the SSC (strong separation condition)), which leads to a generalization of [1]. The self-similar set is completely decomposed into a class of the cylindrical lower (upper) local dimension sets as a coding space is completely decomposed into a class of the lower or upper distribution sets. Using a self-similar measure on the self-similar set, we give a relationship between the distribution sets generated by the frequencies (induced from a self-similar measure) of the codes of the coding space and the cylindrical local dimension sets generated by the cylindrical local dimensions of the self-similar measure. The self-similar measure determining the frequencies gives the information of two necessary axes whereas the axes in [1] are already fixed. In particular, we show that each cylindrical local dimension set is exactly the natural projection of a distribution set having full measure of another self-similar measure related to the distribution set using the strong law of large numbers. The natural projection is a set transformation from a class of the distribution sets of the coding

Received June 2, 2011; Revised August 17, 2011.

2010 Mathematics Subject Classification. Primary 28A78; Secondary 28A80.

Key words and phrases. Hausdorff dimension, packing dimension, self-similar set, distribution set, local dimension set.

This research was supported by Basic Science Research Program through the National Research Foundation of Korea(NRF) funded by the Ministry of Education, Science and Technology(2009-0064869 and 2011-0005114). 
space onto a class of the cylindrical local dimension sets of the self-similar set. This gives essential information of its Hausdorff and packing dimensions. We also give the relation among cylindrical local dimension sets generated by different self-similar measures. In fact, the spectral class by the cylindrical local dimensions of every self-similar measure, except for a singular one, is characterized by the natural projection of the spectral class of the distribution sets. We also compare our results with the recent related ones $[4,5,8,10,11]$. We give some example of the different distribution sets by the differently chosen axes giving the same cylindrical local dimension set. Finally, we provide an essential example to which our results can be applied.

\section{Preliminaries}

Let $\mathbb{N}$ and $\mathbb{R}$ be the set of positive integers and the set of real numbers respectively. An attractor $K$ in the $d$-dimensional Euclidean space $\mathbb{R}^{d}$ of the IFS $\left(f_{1}, \ldots, f_{N}\right)$ of contractions where $N \geq 2$ makes each point $v \in K$ have an infinite sequence $\omega=\left(m_{1}, m_{2}, \ldots\right) \in \Sigma=\{1, \ldots, N\}^{\mathbb{N}}$ where

$$
\{v\}=\bigcap_{n=1}^{\infty} K_{\omega \mid n}
$$

for $K_{\omega \mid n}=K_{m_{1}, \ldots, m_{n}}=f_{m_{1}} \circ \cdots \circ f_{m_{n}}(K)[8] . \omega \mid n$ denotes the truncation of $\omega$ to the $n$th place. In such case, we sometimes write $\pi(\omega)$ for such $v$ using the natural projection $\pi: \Sigma \rightarrow K$ and call $K_{\omega \mid n}$ the cylinder of $v$. We note that $K_{\omega \mid n}$ may be different for the same $v \in K$ since $v$ may have different codes $\omega$. Therefore we write $K_{\omega \mid n}$ for such distinction for the cylinder of $v$. We call such $K_{\omega \mid n}$ the cylinders of $K$ and call $K$ a self-similar set if the IFS $\left(f_{1}, \ldots, f_{N}\right)$ are similitudes.

Each infinite sequence $\omega=\left(m_{1}, m_{2}, \ldots\right)$ in the coding space $\Sigma$ has the unique subset $A\left(x_{n}(\omega)\right)$ of its accumulation points in the simplex of probability vectors in $\mathbb{R}^{N}$ of the vector-valued sequence $\left\{x_{n}(\omega)\right\}=\left\{\left(u_{1}, \ldots, u_{N}\right)_{n}\right\}$ of the probability vectors where $u_{k}$ for $1 \leq k \leq N$ in the probability vector $\left(u_{1}, \ldots, u_{N}\right)_{n}$ for each $n \in \mathbb{N}$ is defined by

$$
u_{k}=\frac{\left|\left\{1 \leq l \leq n: m_{l}=k\right\}\right|}{n} .
$$

The $u_{k}$ for the $n$th place gives the frequency of the digit $k$ in $\omega \mid n=\left(m_{1}, \ldots, m_{n}\right)$. Sometimes we write $n_{k}(\omega \mid n)$ for such $u_{k}$. It is well-known [12] that a set $A\left(x_{n}(\omega)\right)$ of the accumulation points of the vector-valued sequence $\left\{x_{n}(\omega)\right\}$ is a continuum in $\mathbb{R}^{N}$.

For the self-similar measure $\gamma_{\mathbf{p}}$ on $K$ associated with $\mathbf{p}=\left(p_{1}, \ldots, p_{N}\right) \in$ $(0,1)^{N}$ satisfying $\sum_{i=1}^{N} p_{i}=1([4,8,12])$, we write $\underline{E}_{\alpha}^{(\mathbf{p})^{*}}\left(\bar{E}_{\alpha}^{(\mathbf{p})^{*}}\right)$ for the set of points at which the lower (upper) local dimension of $\gamma_{\mathbf{p}}$ on $K$ is exactly $\alpha$, so that

$$
\underline{E}_{\alpha}^{(\mathbf{p})^{*}}=\left\{v \in K: \liminf _{r \rightarrow 0} \frac{\log \gamma_{\mathbf{p}}\left(B_{r}(v)\right)}{\log r}=\alpha\right\},
$$




$$
\bar{E}_{\alpha}^{(\mathbf{p})^{*}}=\left\{v \in K: \limsup _{r \rightarrow 0} \frac{\log \gamma_{\mathbf{p}}\left(B_{r}(v)\right)}{\log r}=\alpha\right\} .
$$

We call $\left\{\underline{E}_{\alpha}^{(\mathbf{p})^{*}}(\neq \phi): \alpha \in \mathbb{R}\right\}$ the spectral class generated by the lower local dimensions of a self-similar measure $\gamma_{\mathbf{p}}$ and $\left\{\bar{E}_{\alpha}^{(\mathbf{p})^{*}}(\neq \phi): \alpha \in \mathbb{R}\right\}$ the spectral class generated by the upper local dimensions of a self-similar measure $\gamma_{\mathbf{p}}$. We call $\alpha$ satisfying $\underline{E}_{\alpha}^{(\mathbf{p})^{*}}(\neq \phi)\left(\bar{E}_{\alpha}^{(\mathbf{p})^{*}}(\neq \phi)\right)$ an associated lower (upper) local dimension of $\gamma_{\mathbf{p}}$. It is well-known $[1,4,12]$ that if the IFS $\left(f_{1}, \ldots, f_{N}\right)$ satisfies the strong separation condition (SSC) then

$$
\begin{aligned}
& \underline{E}_{\alpha}^{(\mathbf{p})^{*}}=\pi\left\{\omega \in \Sigma: \liminf _{n \rightarrow \infty} \frac{\log \gamma_{\mathbf{p}}\left(K_{\omega \mid n}\right)}{\log \left|K_{\omega \mid n}\right|}=\alpha\right\}\left(\equiv \underline{E}_{\alpha}^{(\mathbf{p})}\right), \\
& \bar{E}_{\alpha}^{(\mathbf{p})^{*}}=\pi\left\{\omega \in \Sigma: \limsup _{n \rightarrow \infty} \frac{\log \gamma_{\mathbf{p}}\left(K_{\omega \mid n}\right)}{\log \left|K_{\omega \mid n}\right|}=\alpha\right\}\left(\equiv \bar{E}_{\alpha}^{(\mathbf{p})}\right) .
\end{aligned}
$$

$\left|K_{\omega \mid n}\right|$ denotes the diameter of the cylinder $K_{\omega \mid n}$. In this paper, we assume that the IFS satisfies the open set condition (OSC) $[4,8,12]$ which is a more general condition of the SSC. We mainly discuss the cylindrical local dimension sets $\underline{E}_{\alpha}^{(\mathbf{p})}, \bar{E}_{\alpha}^{(\mathbf{p})}$ instead of $\underline{E}_{\alpha}^{(\mathbf{p})^{*}}, \bar{E}_{\alpha}^{(\mathbf{p})^{*}}$ for studying the self-similar set $K$ of the IFS satisfying the OSC since the cylindrical local dimension sets are quite closely related to the distribution sets. In this paper, we assume that $0 \log 0=0$ for convenience.

Now, we give this cylindrical density theorem for the family of the cylinders of a self-similar set satisfying the OSC which gives the measure separation condition (MSC) in the self-similar set $([7,13])$. From now on, $\operatorname{dim}(E)$ denotes the Hausdorff dimension of $E$ and $\operatorname{Dim}(E)$ denotes the packing dimension of $E([8])$. We note that $\operatorname{dim}(E) \leq \operatorname{Dim}(E)$ for every set $E([8])$.

Proposition 2.1. Let $K_{\omega \mid n}$ be the cylinders of a self-similar set $K$ satisfying the OSC and $\gamma$ be a finite Borel measure on $K$ satisfying $\gamma(\{v \in K: v=$ $\left.\left.\pi(\omega)=\pi\left(\omega^{\prime}\right), \omega \neq \omega^{\prime}\right\}\right)=0$, in particular the self-similar measure on $K$. Let

$$
\begin{aligned}
& \underline{E}_{\leq \alpha} \subset \pi\left\{\omega \in \Sigma: \liminf _{n \rightarrow \infty} \frac{\log \gamma\left(K_{\omega \mid n}\right)}{\log \left|K_{\omega \mid n}\right|} \leq \alpha\right\}, \\
& \underline{E}_{\geq \alpha} \subset \pi\left\{\omega \in \Sigma: \liminf _{n \rightarrow \infty} \frac{\log \gamma\left(K_{\omega \mid n}\right)}{\log \left|K_{\omega \mid n}\right|} \geq \alpha\right\}, \\
& \bar{E}_{\leq \alpha} \subset \pi\left\{\omega \in \Sigma: \limsup _{n \rightarrow \infty} \frac{\log \gamma\left(K_{\omega \mid n}\right)}{\log \left|K_{\omega \mid n}\right|} \leq \alpha\right\}, \\
& \bar{E}_{\geq \alpha} \subset \pi\left\{\omega \in \Sigma: \limsup _{n \rightarrow \infty} \frac{\log \gamma\left(K_{\omega \mid n}\right)}{\log \left|K_{\omega \mid n}\right|} \geq \alpha\right\} .
\end{aligned}
$$

Then we have

(1) $\operatorname{dim}\left(\underline{E}_{\leq \alpha}\right) \leq \alpha$, and if $\gamma\left(\underline{E}_{\geq \alpha}\right)>0$ for a Borel set $\underline{E}_{\geq \alpha}$, then $\operatorname{dim}\left(\underline{E}_{\geq \alpha}\right) \geq$ $\alpha$ 
(2) $\operatorname{Dim}\left(\bar{E}_{\leq \alpha}\right) \leq \alpha$, and if $\gamma\left(\bar{E}_{\geq \alpha}\right)>0$ for a Borel set $\bar{E}_{\geq \alpha}$, then $\operatorname{Dim}\left(\bar{E}_{\geq \alpha}\right)$ $\geq \alpha$.

Further for a Borel set

$$
\underline{E} \subset \pi\left\{\omega \in \Sigma: \liminf _{n \rightarrow \infty} \frac{\log \gamma\left(K_{\omega \mid n}\right)}{\log \left|K_{\omega \mid n}\right|}=\alpha\right\} \equiv \underline{E}_{\alpha},
$$

and for a Borel set

$$
\bar{E} \subset \pi\left\{\omega \in \Sigma: \limsup _{n \rightarrow \infty} \frac{\log \gamma\left(K_{\omega \mid n}\right)}{\log \left|K_{\omega \mid n}\right|}=\alpha\right\} \equiv \bar{E}_{\alpha}
$$

we have

(3) if $\gamma(\underline{E})>0$, then $\operatorname{dim}(\underline{E})=\alpha=\operatorname{dim}\left(\underline{E}_{\alpha}\right)$,

(4) if $\gamma(\overline{\bar{E}})>0$, then $\operatorname{Dim}(\bar{E})=\alpha=\operatorname{Dim}\left(\bar{E}_{\alpha}\right)$.

Proof. We note that $\cup_{n \in \mathbb{N} \cup\{0\}} \cup_{\sigma \in\{1, \ldots, N\}^{n}} \cup_{i \neq j} K_{\sigma i} \cap K_{\sigma j}=\{v \in K: v=$ $\left.\pi(\omega)=\pi\left(\omega^{\prime}\right), \omega \neq \omega^{\prime}\right\}$ and this gives $\gamma\left(\cup_{i \neq j} K_{\sigma i} \cap K_{\sigma j}\right)=0$ for all $\sigma \in$ $\{1, \ldots, N\}^{n}$ where $n \in \mathbb{N} \cup\{0\}$. $\sigma i$ denotes the concatenation of the finite sequence $\sigma$ and $i$. Therefore we can define $\hat{\gamma}$ to be the Borel measure on the coding space $\Sigma$ equipped with the usual ultra-metric topology $([6,9])$ satisfying

$$
\hat{\gamma}(C(\omega \mid n)))=\gamma\left(K_{\omega \mid n}\right),
$$

where the cylinder $C(\omega \mid n)=\{\tau \in \Sigma: \tau|n=\omega| n\}$ for each $\omega \in \Sigma$ and each $n \in \mathbb{N}$ since $\gamma(Z)=0$ where $Z=\left\{v \in K: v=\pi(\omega)=\pi\left(\omega^{\prime}\right), \omega \neq \omega^{\prime}\right\}$. Moreover for the Borel set $E \subset K$, we note that $\hat{\gamma}\left(\pi^{-1}(E)\right)=\gamma(E)$ since $\gamma(Z)=0$. For $E \subset \pi(G)$ where $G \subset \Sigma$, we easily see that if $\gamma(E)>0$, then there exists $F \subset \pi^{-1}(E) \cap G$ such that $\hat{\gamma}(F)>0$ since $\gamma(Z)=0$. Hence it follows from [9, Proposition 1.2].

In particular, the self-similar measure $\gamma_{\mathbf{p}}$ on the self-similar set satisfies the MSC that is $\gamma_{\mathbf{p}}\left(\cup_{i \neq j} K_{i} \cap K_{j}\right)=0([7,13])$. Noting $\gamma_{\mathbf{p}}\left(\cup_{i \neq j} K_{k i} \cap K_{k j}\right)=$ $p_{k} \gamma_{\mathbf{p}}\left(\cup_{i \neq j} K_{i} \cap K_{j}\right)=0$ for all $1 \leq k \leq N$ and continuing these processes, we have $\gamma_{\mathbf{p}}(Z)=0$.

\section{Relation between frequency and density}

From now on, we assume that the similarity ratios of the similarities $\left(f_{1}, \ldots\right.$, $\left.f_{N}\right)$ are $a_{1}, \ldots, a_{N}$ and $K$ is the self-similar set for the IFS $\left(f_{1}, \ldots, f_{N}\right)$ satisfying the OSC and $\gamma_{\mathbf{p}}$ on $K$ is the self-similar measure associated with $\mathbf{p}=\left(p_{1}, \ldots, p_{N}\right) \in(0,1)^{N}$ satisfying $\sum_{k=1}^{N} p_{k}=1$. To avoid the degeneration case, we also assume that $\mathbf{p}=\left(p_{1}, \ldots, p_{N}\right) \neq\left(a_{1}^{s}, \ldots, a_{N}^{s}\right)$ with $\sum_{k=1}^{N} a_{k}^{s}=1$ and $\frac{\log p_{k}}{\log a_{k}}$ is not the same for all $k=1, \ldots, N$. We call the set of the elements $\mathbf{y}=\left(p_{1}, \ldots, p_{N}\right)$ satisfying $\mathbf{y} \in[0,1]^{N}$ and $\sum_{k=1}^{N} y_{k}=1$ the simplex in this paper.

Lemma 3.1. Let $\mathbf{p}=\left(p_{1}, \ldots, p_{N}\right) \in(0,1)^{N}$ with $\sum_{k=1}^{N} p_{k}=1$ and consider $a$ self-similar measure $\gamma_{\mathbf{p}}$ on $K$ and let $\mathbf{r}=\left(r_{1}, \ldots, r_{N}\right) \in[0,1]^{N}$ with $\sum_{k=1}^{N} r_{k}=$ 
1 and

Then

$$
g(\mathbf{r}, \mathbf{p})=\frac{\sum_{k=1}^{N} r_{k} \log p_{k}}{\sum_{k=1}^{N} r_{k} \log a_{k}}
$$

$$
\alpha_{\min } \equiv \min _{1 \leq k \leq N} \frac{\log p_{k}}{\log a_{k}} \leq g(\mathbf{r}, \mathbf{p}) \leq \max _{1 \leq k \leq N} \frac{\log p_{k}}{\log a_{k}} \equiv \alpha_{\max }
$$

Proof. It follows from [8].

We define $g(\mathbf{y}, \mathbf{r})$ for $\mathbf{y}$ and $\mathbf{r}$ as the same manner in the above lemma. The following Lemma $3.2(2)$ is the key idea to explain our multifractal results.

Lemma 3.2. Let $\mathbf{p}=\left(p_{1}, \ldots, p_{N}\right) \in(0,1)^{N}$ with $\sum_{k=1}^{N} p_{k}=1$ and consider $a$ function $\beta(q)$ satisfying $\sum_{k=1}^{N} p_{k}^{q} a_{k}^{\beta(q)}=1$. Given $\alpha_{\min } \leq \alpha \leq \alpha_{\max }$,

(1) when $\alpha \in\left(\alpha_{\min }, \alpha_{\max }\right)$, there exists $q_{0} \in \mathbb{R}$ such that $g(\mathbf{r}, \mathbf{p})=\alpha$ for $\mathbf{r}=\left(r_{1}, \ldots, r_{N}\right)$ where $r_{k}=p_{k}^{q_{0}} a_{k}^{\beta\left(q_{0}\right)}$ such that $\beta^{\prime}\left(q_{0}\right)=-\alpha$, and when $\alpha \in$ $\left\{\alpha_{\min }, \alpha_{\max }\right\}$, there exists a real sequence $\left\{q_{n}\right\}$ such that $g(\mathbf{r}, \mathbf{p})=\alpha$ for $\mathbf{r}=$ $\left(r_{1}, \ldots, r_{N}\right)$ where $r_{k}=\lim _{n \rightarrow \infty} p_{k}^{q_{n}} a_{k}^{\beta\left(q_{n}\right)}$ and $\lim _{n \rightarrow \infty} \beta^{\prime}\left(q_{n}\right)=-\alpha$,

(2) when $\alpha \in\left(\alpha_{\min }, \alpha_{\max }\right)$, if $g(\mathbf{y}, \mathbf{p})=\alpha$ with $\mathbf{y}$ in the simplex, then $g(\mathbf{y}, \mathbf{r})=g(\mathbf{r}, \mathbf{r})$, conversely if $g(\mathbf{y}, \mathbf{r})=g(\mathbf{r}, \mathbf{r})$ with $q_{0} \neq 0$, then $g(\mathbf{y}, \mathbf{p})=$ $\alpha$, and when $\alpha \in\left\{\alpha_{\min }, \alpha_{\max }\right\}$, if $g(\mathbf{y}, \mathbf{p})=\alpha$ with $\mathbf{y}$ in the simplex and $\left\{\frac{\log p_{k}}{\log a_{k}}\right\}_{k=1}^{N}$ are all different, then $g(\mathbf{y}, \mathbf{r})=0$.

Proof. When $\alpha \in\left(\alpha_{\min }, \alpha_{\max }\right)$, (1) follows from (11.35) of [8]. When $\alpha=\alpha_{\max }$, if we put $q_{n}=-n$, then it easily follows. In this case, $r_{k}=\lim _{n \rightarrow \infty} p_{k}^{q_{n}} a_{k}^{\beta\left(q_{n}\right)}$ for each $k$ since $-\alpha_{\min }$ and $-\alpha_{\max }$ are the slopes of the asymptotes of the function $\beta$ ([8]). Similarly, when $\alpha=\alpha_{\min }$, if we put $q_{n}=n$, then it follows. For $(2)$, assume that $g(\mathbf{y}, \mathbf{p})=\alpha$ with $\mathbf{y}$ in the simplex.

When $\alpha \in\left(\alpha_{\min }, \alpha_{\max }\right)$,

$$
\begin{aligned}
g(\mathbf{y}, \mathbf{r}) & =g\left(\mathbf{y},\left(p_{1}^{q_{0}} a_{1}^{\beta\left(q_{0}\right)}, \ldots, p_{N}^{q_{0}} a_{N}^{\beta\left(q_{0}\right)}\right)\right) \\
& =\frac{\sum_{k=1}^{N} y_{k} \log p_{k}^{q_{0}} a_{k}^{\beta\left(q_{0}\right)}}{\sum_{k=1}^{N} y_{k} \log a_{k}} \\
& =q_{0} g(\mathbf{y}, \mathbf{p})+\beta\left(q_{0}\right) \\
& =\alpha q_{0}+\beta\left(q_{0}\right) .
\end{aligned}
$$

Further from (1),

$$
g(\mathbf{r}, \mathbf{p})=\frac{\sum_{k=1}^{N} p_{k}^{q_{0}} a_{k}^{\beta\left(q_{0}\right)} \log p_{k}}{\sum_{k=1}^{N} p_{k}^{q_{0}} a_{k}^{\beta\left(q_{0}\right)} \log a_{k}}=\alpha
$$

This gives

$$
g(\mathbf{r}, \mathbf{r})=g\left(\left(p_{1}^{q_{0}} a_{1}^{\beta\left(q_{0}\right)}, \ldots, p_{N}^{q_{0}} a_{N}^{\beta\left(q_{0}\right)}\right),\left(p_{1}^{q_{0}} a_{1}^{\beta\left(q_{0}\right)}, \ldots, p_{N}^{q_{0}} a_{N}^{\beta\left(q_{0}\right)}\right)\right)
$$




$$
=\frac{\sum_{k=1}^{N} p_{k}^{q_{0}} a_{k}^{\beta\left(q_{0}\right)} \log p_{k}^{q_{0}} a_{k}^{\beta\left(q_{0}\right)}}{\sum_{k=1}^{N} p_{k}^{q_{0}} a_{k}^{\beta\left(q_{0}\right)} \log a_{k}}=\alpha q_{0}+\beta\left(q_{0}\right) .
$$

For the converse, assume that $g(\mathbf{y}, \mathbf{r})=g(\mathbf{r}, \mathbf{r})$. Since $g(\mathbf{r}, \mathbf{r})=\alpha q_{0}+\beta\left(q_{0}\right)$ with $q_{0} \neq 0$,

$$
g(\mathbf{y}, \mathbf{r})=\alpha q_{0}+\beta\left(q_{0}\right)=q_{0} g(\mathbf{y}, \mathbf{p})+\beta\left(q_{0}\right)
$$

gives $g(\mathbf{y}, \mathbf{p})=\alpha$.

When $\alpha \in\left\{\alpha_{\min }, \alpha_{\max }\right\}$, since $\lim _{n \rightarrow \infty} \alpha_{n}=\alpha$ where $\alpha_{n}=-\beta^{\prime}\left(q_{n}\right)$

$$
\begin{aligned}
g(\mathbf{y}, \mathbf{r}) & =\frac{\sum_{k=1}^{N} y_{k} \log \lim _{n \rightarrow \infty}\left[p_{k}^{q_{n}} a_{k}^{\beta\left(q_{n}\right)}\right]}{\sum_{k=1}^{N} y_{k} \log a_{k}} \\
& =\lim _{n \rightarrow \infty}\left[q_{n} g(\mathbf{y}, \mathbf{p})+\beta\left(q_{n}\right)\right] \\
& =\lim _{n \rightarrow \infty}\left[\alpha q_{n}+\beta\left(q_{n}\right)\right] \\
& =\lim _{n \rightarrow \infty}\left[\alpha_{n} q_{n}+\beta\left(q_{n}\right)\right] \\
& =0 .
\end{aligned}
$$

From now on, without specific mention, we fix distinct $i, j$ respectively satisfying

$$
\frac{\log p_{i}}{\log a_{i}}=\min _{1 \leq k \leq N} \frac{\log p_{k}}{\log a_{k}}<\max _{1 \leq k \leq N} \frac{\log p_{k}}{\log a_{k}}=\frac{\log p_{j}}{\log a_{j}} .
$$

It is obvious that there is a unique $\mathbf{z} \in[0,1]^{N}$ for $\mathbf{y}$ in the simplex such that $g(\mathbf{y}, \mathbf{p})=g(\mathbf{z}, \mathbf{p})$ where $\mathbf{z}=\left(z_{1}, \ldots, z_{N}\right)$ with $z_{j}=1-z_{i}$ and $z_{k}=0$ if $k \neq i, j$. We put $z_{\mathbf{y}}=z_{i}$ from now on. More precisely, $z_{\mathbf{y}}$ is the projection of $\mathbf{y}$ in the simplex into the unit interval $[0,1]$ satisfying $z_{\mathbf{y}}=z_{i}$ where $g(\mathbf{y}, \mathbf{p})=g(\mathbf{z}, \mathbf{p})$.

Lemma 3.3. For $\log p_{i} / \log a_{i} \leq \alpha \leq \log p_{j} / \log a_{j}$, we have

$$
\left\{\omega: \max _{\mathbf{y} \in A\left(x_{n}(\omega)\right)} g(\mathbf{y}, \mathbf{p})=\alpha\right\}=\underline{F}(t)
$$

where

$$
\underline{F}(t) \equiv\left\{\omega: \min _{\mathbf{y} \in A\left(x_{n}(\omega)\right)} z_{\mathbf{y}}=t\right\}
$$

and

$$
\frac{t \log p_{i}+(1-t) \log p_{j}}{t \log a_{i}+(1-t) \log a_{j}}=\alpha .
$$

Proof. Assume that $\omega$ satisfies $\max _{\mathbf{y} \in A\left(x_{n}(\omega)\right)} g(\mathbf{y}, \mathbf{p})=\alpha$. Assume that $g(\mathbf{y}, \mathbf{p})$ $=\alpha$ for some $\mathbf{y} \in A\left(x_{n}(\omega)\right)$. Then there is $\mathbf{z} \in[0,1]^{N}$ such that $\alpha=g(\mathbf{y}, \mathbf{p})=$ $g(\mathbf{z}, \mathbf{p})=\frac{t \log p_{i}+(1-t) \log p_{j}}{t \log a_{i}+(1-t) \log a_{j}}$ where $\mathbf{z}=\left(z_{1}, \ldots, z_{N}\right)$ with $z_{j}=1-z_{i}$ and $z_{k}=0$ if $k \neq i, j$ satisfying $z_{i}=z_{\mathbf{y}}=t$. On the other hand, for every $\mathbf{y} \in A\left(x_{n}(\omega)\right), g(\mathbf{y}, \mathbf{p}) \leq \alpha$. Clearly, there is $\mathbf{z} \in[0,1]^{N}$ such that $g(\mathbf{y}, \mathbf{p})=$ $g(\mathbf{z}, \mathbf{p})=\frac{t^{\prime} \log p_{i}+\left(1-t^{\prime}\right) \log p_{j}}{t^{\prime} \log a_{i}+\left(1-t^{\prime}\right) \log a_{j}}$ where $\mathbf{z}=\left(z_{1}, \ldots, z_{N}\right)$ with $z_{j}=1-z_{i}$ and $z_{k}=0$ if $k \neq i, j$ satisfying $z_{i}=z_{\mathbf{y}}=t^{\prime}$. Since $g(\mathbf{y}, \mathbf{p}) \leq \alpha$ and $\frac{\log p_{i}}{\log a_{i}}<\frac{\log p_{j}}{\log a_{j}}$, 
we have $z_{\mathbf{y}}=t^{\prime} \geq t$. This gives $\left\{\omega: \max _{\mathbf{y} \in A\left(x_{n}(\omega)\right)} g(\mathbf{y}, \mathbf{p})=\alpha\right\} \subset \underline{F}(t)$. $\underline{F}(t) \subset\left\{\omega: \max _{\mathbf{y} \in A\left(x_{n}(\omega)\right)} g(\mathbf{y}, \mathbf{p})=\alpha\right\}$ holds from the similar arguments.

The following two theorems generalize Theorem 2 of [1]. Its main idea is that the frequency of a code determines the density of a point for a self-similar measure on a self-similar set and vice versa.

Theorem 3.4. For $\log p_{i} / \log a_{i} \leq \alpha \leq \log p_{j} / \log a_{j}$, we have

$$
\bar{E}_{\alpha}^{(\mathbf{p})}=\pi(\underline{F}(t)),
$$

where

$$
\underline{F}(t)=\left\{\omega: \min _{\mathbf{y} \in A\left(x_{n}(\omega)\right)} z_{\mathbf{y}}=t\right\}
$$

and

$$
\frac{t \log p_{i}+(1-t) \log p_{j}}{t \log a_{i}+(1-t) \log a_{j}}=\alpha .
$$

Proof. From the above lemma, we only need to show that

$$
\begin{aligned}
\bar{E}_{\alpha}^{(\mathbf{p})}=\pi(\{\omega: g(\mathbf{y}, \mathbf{p}) & \leq \alpha \text { for all } \mathbf{y} \in A\left(x_{n}(\omega)\right), \\
g(\mathbf{y}, \mathbf{p}) & \left.\left.=\alpha \text { for some } \mathbf{y} \in A\left(x_{n}(\omega)\right)\right\}\right) .
\end{aligned}
$$

Assume that $\omega$ satisfies $g(\mathbf{y}, \mathbf{p}) \leq \alpha$ for all $\mathbf{y} \in A\left(x_{n}(\omega)\right)$ and $g(\mathbf{y}, \mathbf{p})=\alpha$ for some $\mathbf{y} \in A\left(x_{n}(\omega)\right)$.

Assume that $\mathbf{y}=\left(y_{1}, \ldots, y_{N}\right) \in A\left(x_{n}(\omega)\right)$ such that $g(\mathbf{y}, \mathbf{p})=\alpha$. Since $\mathbf{y}=\lim _{n \rightarrow \infty} x_{k_{n}}(\omega)$ where $x_{k_{n}}(\omega)=\left(n_{1}\left(\omega \mid k_{n}\right), \ldots, n_{N}\left(\omega \mid k_{n}\right)\right)$

$$
\begin{aligned}
\lim _{n \rightarrow \infty} \frac{\log \gamma_{\mathbf{p}}\left(K_{\omega \mid k_{n}}\right)}{\log \left|K_{\omega \mid k_{n}}\right|} & =\lim _{n \rightarrow \infty} \frac{\sum_{l=1}^{N} n_{l}\left(\omega \mid k_{n}\right) \log p_{l}}{\sum_{l=1}^{N} n_{l}\left(\omega \mid k_{n}\right) \log a_{l}} \\
& =\frac{\sum_{l=1}^{N} y_{l} \log p_{l}}{\sum_{l=1}^{N} y_{l} \log a_{l}} \\
& =g(\mathbf{y}, \mathbf{p})=\alpha .
\end{aligned}
$$

Therefore we argue that

$$
\limsup _{k \rightarrow \infty} \frac{\log \gamma_{\mathbf{p}}\left(K_{\omega \mid k}\right)}{\log \left|K_{\omega \mid k}\right|}=\alpha
$$

Otherwise, we may assume that there is a probability vector-valued sequence

$$
\left\{x_{k_{n}}(\omega)\right\}=\left\{\left(n_{1}\left(\omega \mid k_{n}\right), \ldots, n_{N}\left(\omega \mid k_{n}\right)\right)\right\}
$$

such that $\lim _{n \rightarrow \infty} \frac{\log \gamma_{\mathbf{p}}\left(K_{\omega \mid k_{n}}\right)}{\log \left|K_{\omega \mid k_{n}}\right|}>\alpha$. However, $\left\{x_{k_{n}}(\omega)\right\}$ in the simplex which is a compact set gives a limit point $\mathbf{y}=\lim _{m \rightarrow \infty} x_{k_{n_{m}}}(\omega)$ in the simplex satisfying

$$
\lim _{n \rightarrow \infty} \frac{\log \gamma_{\mathbf{p}}\left(K_{\omega \mid k_{n}}\right)}{\log \left|K_{\omega \mid k_{n}}\right|}=\lim _{m \rightarrow \infty} \frac{\log \gamma_{\mathbf{p}}\left(K_{\omega \mid k_{n_{m}}}\right)}{\log \left|K_{\omega \mid k_{n_{m}}}\right|}=g(\mathbf{y}, \mathbf{p})>\alpha
$$


since $\left\{k_{n_{m}}\right\}$ is a subsequence of the sequence $\left\{k_{n}\right\}$. This gives a contradiction since $\omega$ satisfies $g(\mathbf{y}, \mathbf{p}) \leq \alpha$ for all $\mathbf{y} \in A\left(x_{n}(\omega)\right)$ and the limit point $\mathbf{y}=$ $\lim _{m \rightarrow \infty} x_{k_{n_{m}}}(\omega)$ is also a limit point in $A\left(x_{n}(\omega)\right)$.

For the converse, assume that $\pi(\omega) \in \bar{E}_{\alpha}^{(\mathbf{p})}$. Then we see that $\omega$ satisfies $g(\mathbf{y}, \mathbf{p}) \leq \alpha$ for all $\mathbf{y} \in A\left(x_{n}(\omega)\right)$. Otherwise, we may assume that $g(\mathbf{y}, \mathbf{p})>\alpha$ for some $\mathbf{y} \in A\left(x_{n}(\omega)\right)$. Therefore $\mathbf{y}=\lim _{k \rightarrow \infty} x_{n_{k}}(\omega)$ for some subsequence $\left\{n_{k}\right\}$. Hence

$$
\lim _{k \rightarrow \infty} \frac{\log \gamma_{\mathbf{p}}\left(K_{\omega \mid n_{k}}\right)}{\log \left|K_{\omega \mid n_{k}}\right|}=g(\mathbf{y}, \mathbf{p})>\alpha,
$$

which gives a contradiction since our assumption argues that

$$
\limsup _{k \rightarrow \infty} \frac{\log \gamma_{\mathbf{p}}\left(K_{\omega \mid k}\right)}{\log \left|K_{\omega \mid k}\right|}=\alpha .
$$

Now it remains to show that $g(\mathbf{y}, \mathbf{p})=\alpha$ for some $\mathbf{y} \in A\left(x_{n}(\omega)\right)$.

Since $\limsup _{k \rightarrow \infty} \frac{\log \gamma_{\mathbf{p}}\left(K_{\omega \mid k}\right)}{\log \left|K_{\omega \mid k}\right|}=\alpha$, there is a probability vector-valued sequence

$$
\left\{x_{k_{n}}(\omega)\right\}=\left\{\left(n_{1}\left(\omega \mid k_{n}\right), \ldots, n_{N}\left(\omega \mid k_{n}\right)\right)\right\}
$$

such that $\lim _{n \rightarrow \infty} \frac{\log \gamma_{\mathbf{p}}\left(K_{\omega \mid k_{n}}\right)}{\log \left|K_{\omega \mid k_{n}}\right|}=\alpha$. Using the similar arguments above, we find a limit point $\mathbf{y}=\lim _{m \rightarrow \infty} x_{k_{n_{m}}}(\omega)$ in the simplex satisfying

$$
\lim _{n \rightarrow \infty} \frac{\log \gamma_{\mathbf{p}}\left(K_{\omega \mid k_{n}}\right)}{\log \left|K_{\omega \mid k_{n}}\right|}=\lim _{m \rightarrow \infty} \frac{\log \gamma_{\mathbf{p}}\left(K_{\omega \mid k_{n_{m}}}\right)}{\log \left|K_{\omega \mid k_{n_{m}}}\right|}=g(\mathbf{y}, \mathbf{p})=\alpha
$$

since $\left\{k_{n_{m}}\right\}$ is a subsequence of the sequence $\left\{k_{n}\right\}$.

The following is the dual result of the above theorem. We omit its proof.

Theorem 3.5. For $\log p_{i} / \log a_{i} \leq \alpha \leq \log p_{j} / \log a_{j}$, we have

$$
\underline{E}_{\alpha}^{(\mathbf{p})}=\pi(\bar{F}(t)),
$$

where

$$
\bar{F}(t) \equiv\left\{\omega: \max _{\mathbf{y} \in A\left(x_{n}(\omega)\right)} z_{\mathbf{y}}=t\right\}
$$

and

$$
\frac{t \log p_{i}+(1-t) \log p_{j}}{t \log a_{i}+(1-t) \log a_{j}}=\alpha .
$$

Remark 3.6. We note that $A\left(x_{n}(\omega)\right) \neq \phi$ for every $\omega$ and

$$
\begin{aligned}
\pi(\{\omega: g(\mathbf{y}, \mathbf{p}) & \geq \alpha \text { for all } \mathbf{y} \in A\left(x_{n}(\omega)\right), \\
g(\mathbf{y}, \mathbf{p}) & \left.\left.=\alpha \text { for some } \mathbf{y} \in A\left(x_{n}(\omega)\right)\right\}\right)=\pi(\bar{F}(t))=\underline{E}_{\alpha}^{(\mathbf{p})} .
\end{aligned}
$$

Theorem 3.7. For $\log p_{i} / \log a_{i} \leq \alpha \leq \log p_{j} / \log a_{j}$, we have

$$
E_{\alpha}^{(\mathbf{p})}=\pi(F(t))
$$


where

$$
F(t) \equiv\left\{\omega: \min _{\mathbf{y} \in A\left(x_{n}(\omega)\right)} z_{\mathbf{y}}=\max _{\mathbf{y} \in A\left(x_{n}(\omega)\right)} z_{\mathbf{y}}=t\right\}
$$

and

$$
\frac{t \log p_{i}+(1-t) \log p_{j}}{t \log a_{i}+(1-t) \log a_{j}}=\alpha .
$$

Proof. By the above theorems, we see that $\pi(F(t))=\pi(\underline{F}(t) \cap \bar{F}(t)) \subset \pi(\underline{F}(t)) \cap$ $\pi(\bar{F}(t))=\bar{E}_{\alpha}^{(\mathbf{p})} \cap \underline{E}_{\alpha}^{(\mathbf{p})}=E_{\alpha}^{(\mathbf{p})} . E_{\alpha}^{(\mathbf{p})} \subset \pi(F(t))$ follows similarly from the proof of Theorem 3.4 and its dual proof of Theorem 3.5.

Remark 3.8. We call the above $\underline{F}(t)(\bar{F}(t))$ the lower (upper) distribution set (induced from $(\mathbf{p}, i, j, t))$ and $F(t)$ the distribution set (induced from $(\mathbf{p}, i, j, t)$ ). We call such $(i, j)$ axes the parameter axes and call such distribution sets the parameter distribution sets for the self-similar measure $\gamma_{\mathbf{p}}$ with respect to the parameter axes.

Remark 3.9. If $\min _{1 \leq k \leq N} \frac{\log p_{k}}{\log a_{k}}=\max _{1 \leq k \leq N} \frac{\log p_{k}}{\log a_{k}}$, then we have

$$
\bar{E}_{\alpha}^{(\mathbf{p})}=\underline{E}_{\alpha}^{(\mathbf{p})}=E_{\alpha}^{(\mathbf{p})}=K
$$

for

$$
\alpha=\min _{1 \leq k \leq N} \frac{\log p_{k}}{\log a_{k}}=\max _{1 \leq k \leq N} \frac{\log p_{k}}{\log a_{k}} .
$$

Remark 3.10. We have

$$
K=\bigcup_{\frac{\log p_{i}}{\log a_{i}} \leq \alpha \leq \frac{\log p_{j}}{\log a_{j}}} \bar{E}_{\alpha}^{(\mathbf{p})}=\bigcup_{0 \leq t \leq 1} \pi(\underline{F}(t)) .
$$

Similarly we have

$$
K=\bigcup_{\frac{\log p_{i}}{\log a_{i}} \leq \alpha \leq \frac{\log p_{j}}{\log a_{j}}} \underline{E}_{\alpha}^{(\mathbf{p})}=\bigcup_{0 \leq t \leq 1} \pi(\bar{F}(t)) .
$$

\section{Subset relation and multifractal spectrum}

The following theorems are byproducts of our main results Theorems 3.4 and 3.5. They generalize the multifractal results for the self-similar set of [8] and give the relation between our results and those of [8]. They are also generalizations of Corollaries 5 and 7 with Theorem 6 of [1]. In the following theorems, let $t_{0}$ be the real number satisfying

$$
\frac{t_{0} \log p_{i}+\left(1-t_{0}\right) \log p_{j}}{t_{0} \log a_{i}+\left(1-t_{0}\right) \log a_{j}}=g\left(\mathbf{r}_{0}, \mathbf{p}\right)
$$

for $\mathbf{r}_{0}=\left(a_{1}^{s}, \ldots, a_{N}^{s}\right)$ with $\sum_{k=1}^{N} a_{k}^{s}=1$. We note that when $\alpha \in\left(\alpha_{\min }, \alpha_{\max }\right)$, there exists $q_{0} \in \mathbb{R}$ such that $g(\mathbf{r}, \mathbf{p})=\alpha$ for $\mathbf{r}=\left(r_{1}, \ldots, r_{N}\right)$ where $r_{k}=$ $p_{k}^{q_{0}} a_{k}^{\beta\left(q_{0}\right)}$ such that $\beta^{\prime}\left(q_{0}\right)=-\alpha$ by Lemma 3.2(1). Therefore in the following 
theorems, given $0<t<1$, we put $\mathbf{r}=\mathbf{r}(t)=\left(r_{1}, \ldots, r_{N}\right)$ satisfying $r_{k}=$ $p_{k}^{q_{0}} a_{k}^{\beta\left(q_{0}\right)}$ for $\beta^{\prime}\left(q_{0}\right)=-\alpha$ where

$$
\alpha=\alpha(t)=\frac{t \log p_{i}+(1-t) \log p_{j}}{t \log a_{i}+(1-t) \log a_{j}} .
$$

Theorem 4.1. We have the following:

(1) if $0<t_{0}<t<1$, then

$$
\bar{E}_{\alpha}^{(\mathbf{p})}=\pi(\underline{F}(t))=\bar{E}_{g(\mathbf{r}(t), \mathbf{r}(t))}^{(\mathbf{r}(t))},
$$

(2) if $0<t<t_{0}<1$, then

$$
\bar{E}_{\alpha}^{(\mathbf{p})}=\pi(\underline{F}(t))=\underline{E}_{g(\mathbf{r}(t), \mathbf{r}(t))}^{(\mathbf{r}(t))},
$$

(3) if $0<t_{0}<t<1$, then

$$
\underline{E}_{\alpha}^{(\mathbf{p})}=\pi(\bar{F}(t))=\underline{E}_{g(\mathbf{r}(t), \mathbf{r}(t))}^{(\mathbf{r}(t))},
$$

(4) if $0<t<t_{0}<1$, then

$$
\underline{E}_{\alpha}^{(\mathbf{p})}=\pi(\bar{F}(t))=\bar{E}_{g(\mathbf{r}(t), \mathbf{r}(t))}^{(\mathbf{r}(t))} .
$$

Proof. If $0<t<1$, then there exists $q_{0} \in \mathbb{R}$ satisfying $\beta^{\prime}\left(q_{0}\right)=-\alpha$ since

$$
\alpha_{\min }<\alpha=\frac{t \log p_{i}+(1-t) \log p_{j}}{t \log a_{i}+(1-t) \log a_{j}}<\alpha_{\max } .
$$

For $\mathbf{r}=\mathbf{r}(t)=\left(r_{1}, \ldots, r_{N}\right)$ such that $r_{k}=p_{k}^{q_{0}} a_{k}^{\beta\left(q_{0}\right)}$ satisfying $\beta^{\prime}\left(q_{0}\right)=-\alpha$, we have

$$
\begin{aligned}
g(\mathbf{y}, \mathbf{r}) & =g\left(\mathbf{y},\left(p_{1}^{q_{0}} a_{1}^{\beta\left(q_{0}\right)}, \ldots, p_{N}^{q_{0}} a_{N}^{\beta\left(q_{0}\right)}\right)\right) \\
& =\frac{\sum_{k=1}^{N} y_{k} \log p_{k}^{q_{0}} a_{k}^{\beta\left(q_{0}\right)}}{\sum_{k=1}^{N} y_{k} \log a_{k}} \\
& =q_{0} g(\mathbf{y}, \mathbf{p})+\beta\left(q_{0}\right),
\end{aligned}
$$

where $\mathbf{y}=\left(y_{1}, \ldots, y_{N}\right)$ in the simplex. Therefore $g(\mathbf{y}, \mathbf{r}) \leq \alpha q_{0}+\beta\left(q_{0}\right)$ for $g(\mathbf{y}, \mathbf{p}) \leq \alpha$ with $q_{0} \geq 0$. Similarly $g(\mathbf{y}, \mathbf{r}) \geq \alpha q_{0}+\beta\left(q_{0}\right)$ for $g(\mathbf{y}, \mathbf{p}) \leq \alpha$ with $q_{0} \leq 0$.

We note that

$$
0<t_{0} \leq t<1 \Longleftrightarrow q_{0} \geq 0
$$

and

$$
0<t \leq t_{0}<1 \Longleftrightarrow q_{0} \leq 0
$$

We also note that

$$
t=t_{0} \Longleftrightarrow q_{0}=0
$$

For $q_{0} \geq 0$,

$$
\pi(\underline{F}(t)) \subset \bar{E}_{g(\mathbf{r}(t), \mathbf{r}(t))}^{(\mathbf{r}(t))} .
$$


Precisely, if $v \in \pi(\underline{F}(t))$, then $v=\pi(\omega)$ with $g(\mathbf{y}, \mathbf{p}) \leq \alpha$ for all $\mathbf{y} \in A\left(x_{n}(\omega)\right)$, and $g(\mathbf{y}, \mathbf{p})=\alpha$ for some $\mathbf{y} \in A\left(x_{n}(\omega)\right)$ since

$$
\left\{\omega: \max _{\mathbf{y} \in A\left(x_{n}(\omega)\right)} g(\mathbf{y}, \mathbf{p})=\alpha\right\}=\underline{F}(t)
$$

by Lemma 3.3. Since $q_{0} \geq 0$, we have $g(\mathbf{y}, \mathbf{r}) \leq \alpha q_{0}+\beta\left(q_{0}\right)=g(\mathbf{r}(t), \mathbf{r}(t))$ for all $\mathbf{y} \in A\left(x_{n}(\omega)\right)$, and $g(\mathbf{y}, \mathbf{r})=\alpha q_{0}+\beta\left(q_{0}\right)=g(\mathbf{r}(t), \mathbf{r}(t))$ for some $\mathbf{y} \in A\left(x_{n}(\omega)\right)$. Let

$$
G=\left\{\omega: \max _{\mathbf{y} \in A\left(x_{n}(\omega)\right)} g(\mathbf{y}, \mathbf{r})=g(\mathbf{r}(t), \mathbf{r}(t))\right\} .
$$

Then $\pi(G)=\bar{E}_{g(\mathbf{r}(t), \mathbf{r}(t))}^{(\mathbf{r}(t))}$ by the similar arguments of the proof of Theorem 3.4. Therefore $\omega \in G$. This gives $v=\pi(\omega) \in \bar{E}_{g(\mathbf{r}(t), \mathbf{r}(t))}^{(\mathbf{r}(t)}$.

For (1), since $\bar{E}_{\alpha}^{(\mathbf{p})}=\pi(\underline{F}(t))$ from Theorem 3.4, we only need to show that $\bar{E}_{g(\mathbf{r}(t), \mathbf{r}(t))}^{(\mathbf{r}(t))} \subset \bar{E}_{\alpha}^{(\mathbf{p})}$. If $v \in \pi(G)=\bar{E}_{g(\mathbf{r}(t), \mathbf{r}(t))}^{(\mathbf{r}(t)}$, then $v=\pi(\omega)$ with $g(\mathbf{y}, \mathbf{r}) \leq \alpha q_{0}+\beta\left(q_{0}\right)=g(\mathbf{r}(t), \mathbf{r}(t))$ for all $\mathbf{y} \in A\left(x_{n}(\omega)\right)$, and $g(\mathbf{y}, \mathbf{r})=$ $\alpha q_{0}+\beta\left(q_{0}\right)=g(\mathbf{r}(t), \mathbf{r}(t))$ for some $\mathbf{y} \in A\left(x_{n}(\omega)\right)$. Since $q_{0}>0$ and $g(\mathbf{y}, \mathbf{r})=$ $q_{0} g(\mathbf{y}, \mathbf{p})+\beta\left(q_{0}\right)$, we have $g(\mathbf{y}, \mathbf{p}) \leq \alpha$ for all $\mathbf{y} \in A\left(x_{n}(\omega)\right)$ and $g(\mathbf{y}, \mathbf{p})=\alpha$ for some $\mathbf{y} \in A\left(x_{n}(\omega)\right)$. This gives $v=\pi(\omega) \in \pi(\underline{F}(t))=\bar{E}_{\alpha}^{(\mathbf{p})}$. We have (2), (3) and (4) from the similar arguments above.

Theorem 4.2. We have the following:

(1) if $0<t_{0} \leq t<1$, then

$$
\operatorname{dim}(\pi(\underline{F}(t)))=\operatorname{Dim}(\pi(\underline{F}(t)))=g(\mathbf{r}(t), \mathbf{r}(t))=\alpha q_{0}+\beta\left(q_{0}\right),
$$

hence

$$
\operatorname{dim}\left(\bar{E}_{\alpha(t)}^{(\mathbf{p})}\right)=\operatorname{Dim}\left(\bar{E}_{\alpha(t)}^{(\mathbf{p})}\right)=g(\mathbf{r}(t), \mathbf{r}(t))=\alpha q_{0}+\beta\left(q_{0}\right),
$$

(2) if $0<t \leq t_{0}<1$, then

$$
\operatorname{dim}(\pi(\bar{F}(t)))=\operatorname{Dim}(\pi(\bar{F}(t)))=g(\mathbf{r}(t), \mathbf{r}(t))=\alpha q_{0}+\beta\left(q_{0}\right),
$$

hence

$$
\operatorname{dim}\left(\underline{E}_{\alpha(t)}^{(\mathbf{p})}\right)=\operatorname{Dim}\left(\underline{E}_{\alpha(t)}^{(\mathbf{p})}\right)=g(\mathbf{r}(t), \mathbf{r}(t))=\alpha q_{0}+\beta\left(q_{0}\right) .
$$

Proof. Since $\left\{\omega: A\left(x_{n}(\omega)\right)=\{\mathbf{r}(t)\}\right\} \subset \underline{F}(t) \cap \bar{F}(t)=F(t)$, we have

$$
\gamma_{\mathbf{r}(t)}(\pi(F(t)))=1
$$

from the strong law of large numbers. Noting that $\gamma$ is a self-similar measure on the self-similar set, we have (1) and (2) from Proposition 2.1 with the following arguments. For the arguments, we also note that the cylindrical local dimension sets correspond with the natural projection of the distribution sets from Theorems 3.4 and 3.5 .

For (1), $q_{0} \geq 0$ gives

$$
\bar{E}_{\alpha}^{(\mathbf{p})}=\pi(\underline{F}(t)) \subset \bar{E}_{g(\mathbf{r}(t), \mathbf{r}(t))}^{(\mathbf{r}(t))}
$$


and

$$
\underline{E}_{\alpha}^{(\mathbf{p})}=\pi(\bar{F}(t)) \subset \underline{E}_{g(\mathbf{r}(t), \mathbf{r}(t))}^{(\mathbf{r}(t))} .
$$

Since $\pi(F(t)) \subset \pi(\underline{F}(t)) \subset \bar{E}_{g(\mathbf{r}(t), \mathbf{r}(t))}^{(\mathbf{r}(t))}$ and $\gamma_{\mathbf{r}(t)}(\pi(F(t)))=1$, we have $g(\mathbf{r}(t), \mathbf{r}(t)) \leq \operatorname{dim}(\pi(F(t))) \leq \operatorname{dim}(\pi(\underline{F}(t))) \leq \operatorname{Dim}(\pi(\underline{F}(t))) \leq g(\mathbf{r}(t), \mathbf{r}(t))$

from Proposition 2.1.

For $(2), q_{0} \leq 0$ gives

$$
\underline{E}_{\alpha}^{(\mathbf{p})}=\pi(\bar{F}(t)) \subset \bar{E}_{g(\mathbf{r}(t), \mathbf{r}(t))}^{(\mathbf{r}(t))}
$$

and

$$
\bar{E}_{\alpha}^{(\mathbf{p})}=\pi(\underline{F}(t)) \subset \underline{E}_{g(\mathbf{r}(t), \mathbf{r}(t))}^{(\mathbf{r}(t))} .
$$

Since $\pi(F(t)) \subset \pi(\bar{F}(t)) \subset \bar{E}_{g(\mathbf{r}(t), \mathbf{r}(t))}^{(\mathbf{r}(t))}$ and $\gamma_{\mathbf{r}(t)}(\pi(F(t)))=1$, we have

$$
g(\mathbf{r}(t), \mathbf{r}(t)) \leq \operatorname{dim}(\pi(F(t))) \leq \operatorname{dim}(\pi(\bar{F}(t))) \leq \operatorname{Dim}(\pi(\bar{F}(t))) \leq g(\mathbf{r}(t), \mathbf{r}(t))
$$

from Proposition 2.1.

Theorem 4.3. We have the following:

(1) if $0<t \leq t_{0}<1$, then

$$
\operatorname{dim}(\pi(\underline{F}(t)))=g(\mathbf{r}(t), \mathbf{r}(t))=\alpha q_{0}+\beta\left(q_{0}\right),
$$

and

$$
\operatorname{Dim}(\pi(\underline{F}(t)))=s,
$$

hence

$$
\operatorname{dim}\left(\bar{E}_{\alpha(t)}^{(\mathbf{p})}\right)=g(\mathbf{r}(t), \mathbf{r}(t))=\alpha q_{0}+\beta\left(q_{0}\right),
$$

and

$$
\operatorname{Dim}\left(\bar{E}_{\alpha(t)}^{(\mathbf{p})}\right)=s,
$$

(2) if $0<t_{0} \leq t<1$, then

$$
\operatorname{dim}(\pi(\bar{F}(t)))=g(\mathbf{r}(t), \mathbf{r}(t))=\alpha q_{0}+\beta\left(q_{0}\right),
$$

and

hence

$$
\operatorname{Dim}(\pi(\bar{F}(t)))=s,
$$

and

$$
\operatorname{dim}\left(\underline{E}_{\alpha(t)}^{(\mathbf{p})}\right)=g(\mathbf{r}(t), \mathbf{r}(t))=\alpha q_{0}+\beta\left(q_{0}\right),
$$

$$
\operatorname{Dim}\left(\underline{E}_{\alpha(t)}^{(\mathbf{p})}\right)=s .
$$

Proof. We use the arguments in the above proof. For (1), $q_{0} \leq 0$ gives

$$
\bar{E}_{\alpha}^{(\mathbf{p})}=\pi(\underline{F}(t)) \subset \underline{E}_{g(\mathbf{r}(t), \mathbf{r}(t))}^{(\mathbf{r}(t))} .
$$

Since $\pi(F(t)) \subset \pi(\underline{F}(t)) \subset \underline{E}_{g(\mathbf{r}(t), \mathbf{r}(t))}^{(\mathbf{r}(t))}$ and $\gamma_{\mathbf{r}(t)}(\pi(F(t)))=1$, we have

$$
g(\mathbf{r}(t), \mathbf{r}(t)) \leq \operatorname{dim}(\pi(F(t))) \leq \operatorname{dim}(\pi(\underline{F}(t))) \leq g(\mathbf{r}(t), \mathbf{r}(t))
$$

from Proposition 2.1. 
For $(2), q_{0} \geq 0$ gives

$$
\underline{E}_{\alpha}^{(\mathbf{p})}=\pi(\bar{F}(t)) \subset \underline{E}_{g(\mathbf{r}(t), \mathbf{r}(t))}^{(\mathbf{r}(t))} .
$$

Since $\pi(F(t)) \subset \pi(\bar{F}(t)) \subset \underline{E}_{g(\mathbf{r}(t), \mathbf{r}(t))}^{(\mathbf{r}(t))}$ and $\gamma_{\mathbf{r}(t)}(\pi(F(t)))=1$, we have

$$
g(\mathbf{r}(t), \mathbf{r}(t)) \leq \operatorname{dim}(\pi(F(t))) \leq \operatorname{dim}(\pi(\bar{F}(t))) \leq g(\mathbf{r}(t), \mathbf{r}(t))
$$

from Proposition 2.1. For the arguments of the packing dimension of (1) and (2), we note that the distribution set of (1) or (2) contains $\left\{\omega: A\left(x_{n}(\omega)\right)=\mathbf{C}\right\}$ where $\mathbf{r}_{\mathbf{0}} \in \mathbf{C}$ and $\mathbf{C}$ is a continuum. It follows from that the packing dimension of $\pi\left(\left\{\omega: A\left(x_{n}(\omega)\right)=\mathbf{C}\right\}\right)$ is $s([4])$ where $\sum_{k=1}^{N} a_{k}^{s}=1$.

Theorem 4.4. We have the following if $\left\{\frac{\log p_{k}}{\log a_{k}}\right\}_{k=1}^{N}$ are all different:

(1) $\operatorname{dim}(\pi(\underline{F}(1)))=\operatorname{Dim}(\pi(\underline{F}(1)))=\operatorname{dim}\left(\bar{E}_{\alpha(1)}^{(\mathbf{p})}\right)=\operatorname{Dim}\left(\bar{E}_{\alpha(1)}^{(\mathbf{p})}\right)=0$,

(2) $\operatorname{dim}(\pi(\underline{F}(0)))=\operatorname{dim}\left(\bar{E}_{\alpha(0)}^{(\mathbf{p})}\right)=0, \operatorname{Dim}(\pi(\underline{F}(0)))=\operatorname{Dim}\left(\bar{E}_{\alpha(0)}^{(\mathbf{p})}\right)=s$,

(3) $\operatorname{dim}(\pi(\bar{F}(1)))=\operatorname{dim}\left(\underline{E}_{\alpha(1)}^{(\mathbf{p})}\right)=0, \operatorname{Dim}(\pi(\bar{F}(1)))=\operatorname{Dim}\left(\underline{E}_{\alpha(1)}^{(\mathbf{p})}\right)=s$,

(4) $\operatorname{dim}(\pi(\bar{F}(0)))=\operatorname{Dim}(\pi(\bar{F}(0)))=\operatorname{dim}\left(\underline{E}_{\alpha(0)}^{(\mathbf{p})}\right)=\operatorname{Dim}\left(\underline{E}_{\alpha(0)}^{(\mathbf{p})}\right)=0$.

Proof. It easily follows from Lemma 3.2(2) for the case $\alpha \in\left\{\alpha_{\min }, \alpha_{\max }\right\}$ and Theorems 3.4 and 3.5 and the similar arguments of the packing dimension of (1) and (2) of the above proof.

Remark 4.5. Every number $t \in[0,1]$ defines a point $\mathbf{z}$ in the edge lying on the two dimensional $(i, j)$-plane in $\mathbb{R}^{N}$ of the simplex $S$ and $\mathbf{z}$ also defines a hyper-plane $\{\mathbf{y} \in S: g(\mathbf{y}, \mathbf{p})=\alpha\}$ of the simplex $S$ for some $\alpha$ where the intersection of the edge and the hyper-plane is $\mathbf{z}$. In the proofs of the above theorems, in particular, if $t=t_{0}$, then $q_{0}=0$ and it gives $\mathbf{r}(t)=\mathbf{r}_{0}=$ $\left(a_{1}^{s}, \ldots, a_{N}^{s}\right)$ with $\sum_{k=1}^{N} a_{k}^{s}=1$, so $E_{s}^{\left(\mathbf{r}_{0}\right)}=K$. We also note that the above cylindrical multifractal results provide us with a new definition (Example 2) of the generalized Riesz-Nágy -Takács function and a generalization [3] of the results [2] of the derivative of the Riesz-Nágy-Takács function.

Remark 4.6. We note that for every $\alpha$, unless $\min _{1 \leq k \leq N} \frac{\log p_{k}}{\log a_{k}}=\max _{1 \leq k \leq N} \frac{\log p_{k}}{\log a_{k}}$,

$$
\operatorname{dim}\left(\bar{E}_{\alpha}^{(\mathbf{p})}\right)=\operatorname{dim}\left(E_{\alpha}^{(\mathbf{p})}\right)=\operatorname{Dim}\left(E_{\alpha}^{(\mathbf{p})}\right)=\operatorname{dim}\left(\underline{E}_{\alpha}^{(\mathbf{p})}\right),
$$

even though there exists $\alpha$ such that $\operatorname{Dim}\left(\underline{E}_{\alpha}^{(\mathbf{p})}\right) \neq \operatorname{Dim}\left(E_{\alpha}^{(\mathbf{p})}\right)$ or $\operatorname{Dim}\left(E_{\alpha}^{(\mathbf{p})}\right) \neq$ $\operatorname{Dim}\left(\bar{E}_{\alpha}^{(\mathbf{p})}\right)$.

Remark 4.7. In the above cases for the OSC, we use the cylindrical density theorem (Proposition 2.1) for the family of the cylinders of the self-similar set $K$ instead of the Frostman's density theorem [8]. In the above cases for the SSC, our cylindrical local dimension set is exactly the local dimension set[8], so we may use the Frostman's density theorem instead of the cylindrical density theorem (Proposition 2.1). Further, our results of [1] are also direct 
consequences of Theorem 4.2 for the self-similar set $K$ with $N=2$ satisfying the SSC.

Example 1. Let $K=[0,1]$ be the self-similar set for the IFS $\left(f_{1}, f_{2}, f_{3}\right)$ satisfying the OSC whose similarity ratios are $\left(a_{1}, a_{2}, a_{3}\right)=(1 / 9,5 / 9,1 / 3)$ and $\gamma_{\mathbf{p}}$ on $K$ be the self-similar measure associated with $\mathbf{p}=\left(p_{1}, p_{2}, p_{3}\right)=$ $(1 / 4,1 / 4,1 / 2)$. We can consider two different distribution structures fixing distinct $i, j$ respectively

$$
\frac{\log p_{i}}{\log a_{i}}=\min _{1 \leq k \leq 3} \frac{\log p_{k}}{\log a_{k}}=\frac{\log 2}{\log 3}=\frac{\log 4}{\log 9}<\frac{\log 4}{\log 9 / 5}=\max _{1 \leq k \leq 3} \frac{\log p_{k}}{\log a_{k}}=\frac{\log p_{j}}{\log a_{j}} .
$$

For $(i, j)=(1,2)$, in Theorem $4.1, t_{0}$ is the solution of the equation

$$
\frac{t_{0} \log 1 / 4+\left(1-t_{0}\right) \log 1 / 4}{t_{0} \log 1 / 9+\left(1-t_{0}\right) \log 5 / 9}=g\left(\mathbf{r}_{0}, \mathbf{p}\right)
$$

for $\mathbf{r}_{0}=\left(a_{1}, a_{2}, a_{3}\right)=(1 / 9,5 / 9,1 / 3)$.

Similarly, for $(i, j)=(3,2)$, in Theorem $4.1, T_{0}$ is the solution of the equation

$$
\frac{T_{0} \log 1 / 2+\left(1-T_{0}\right) \log 1 / 4}{T_{0} \log 1 / 3+\left(1-T_{0}\right) \log 5 / 9}=g\left(\mathbf{r}_{0}, \mathbf{p}\right)
$$

for $\mathbf{r}_{0}=\left(a_{1}, a_{2}, a_{3}\right)=(1 / 9,5 / 9,1 / 3)$. We note that the solutions $t_{0}$ and $T_{0}$ of the two different equations are different, which gives the different distribution sets. More precisely, given $\frac{\log 4}{\log 9}<\alpha<\frac{\log 4}{\log 9 / 5}$, we find different $t$ and $T$ for $(i, j)=(1,2)$ and $(i, j)=(3,2)$ cases respectively such that $\alpha=\alpha(t)$ and $\alpha=\alpha(T)$ in Theorem 4.1. However we note that the different distribution sets give the same cylindrical local dimension set in Theorem 4.1. For example, in Theorem 4.1(1),

$$
\bar{E}_{\alpha}^{(\mathbf{p})}=\pi(\underline{F}(t))=\pi(\underline{G}(T))=\bar{E}_{g(\mathbf{r}, \mathbf{r})}^{(\mathbf{r})},
$$

where $\mathbf{r}=\left(r_{1}, r_{2}, r_{3}\right)$ satisfying $r_{k}=p_{k}^{q_{0}} a_{k}^{\beta\left(q_{0}\right)}$ for $\beta^{\prime}\left(q_{0}\right)=-\alpha$ where

$$
\alpha=\frac{t \log p_{1}+(1-t) \log p_{2}}{t \log a_{1}+(1-t) \log a_{2}}=\frac{T \log p_{3}+(1-T) \log p_{2}}{T \log a_{3}+(1-T) \log a_{2}}
$$

for $0<t_{0}<t<1$ and $0<T_{0}<T<1$. We note that $\underline{F}(t)$ is the lower distribution set induced from $(\mathbf{p}, 1,2, t)$ and $\underline{G}(T)$ is the lower distribution set induced from $(\mathbf{p}, 3,2, T)$. This example shows that the choice of the parameter $\operatorname{axes}(=(i, j)$ axes $)$ is not unique.

Example $2([3])$. For the probability vectors $\left(a_{1}, \ldots, a_{N}\right) \in(0,1)^{N}$ and $\mathbf{p}=$ $\left(p_{1}, \ldots, p_{N}\right) \in(0,1)^{N}$ where $N \geq 2$ is a positive integer,

$$
[0,1]=\bigcup_{k=1}^{N} f_{k}([0,1]),
$$


where $f_{k}(x)=a_{k} x+\sum_{i=1}^{k-1} a_{i}$, and $\gamma_{\mathbf{p}}$ is the unique probability measure on $[0,1]$ such that

$$
\gamma_{\mathbf{p}}=\sum_{i=1}^{N} p_{i} \gamma_{\mathbf{p}} \circ f_{i}^{-1}
$$

We define

$$
S(x)=\gamma_{\mathbf{p}}([0, x])
$$

where $\gamma_{\mathbf{p}}$ is the self-similar measure on the self-similar set $[0,1]$. We call the function $S$ the generalized Riesz-Nágy-Takács function (GRNT function). We assume that $\mathbf{p}=\left(p_{1}, \ldots, p_{N}\right) \neq\left(a_{1}, \ldots, a_{N}\right)$. Let $t_{1}$ be the real number satisfying

$$
\frac{t_{1} \log p_{i}+\left(1-t_{1}\right) \log p_{j}}{t_{1} \log a_{i}+\left(1-t_{1}\right) \log a_{j}}=1 .
$$

Then there is $q$ satisfying $\beta^{\prime}(q)=-1$. Then $\mathbf{r}_{1}=\left(p_{1}^{q} a_{1}^{\beta(q)}, \ldots, p_{N}^{q} a_{N}^{\beta(q)}\right)$ satisfies $g\left(\mathbf{r}_{1}, \mathbf{p}\right)=1$. For the non-differentiability points $M$ of the GRNT function $S$, using a variation of Theorem 4.3, we have

$$
0<g\left(\mathbf{r}_{1}, \mathbf{r}_{1}\right) \leq \operatorname{dim}(M) \leq \operatorname{Dim}(M)=1 .
$$

\section{References}

[1] I.-S. Baek, Relation between spectral classes of a self-similar Cantor set, J. Math. Anal. Appl. 292 (2004), no. 1, 294-302.

[2] $\longrightarrow$, Derivative of the Riesz-Nágy - Takács function, Bull. Korean Math. Soc. 48 (2011), no. 2, 261-275.

[3] — The derivative and moment of the generalized Riesz-Nágy-Takács function, preprint.

[4] I.-S. Baek, L. Olsen, and N. Snigireva, Divergence points of self-similar measures and packing dimension, Adv. Math. 214 (2007), no. 1, 267-287.

[5] R. Cawley and R. D. Mauldin, Multifractal decompositions of Moran fractals, Adv. Math. 92 (1992), no. 2, 196-236.

[6] G. A. Edgar, Measure, Topology, and Fractal Geometry, Springer Verlag, 1990.

[7] M. Elekes, T. Keleti, and A. Máthé, Self-similar and self-affine sets; measures of the intersection of two copies, Ergodic Theory Dynam. Systems 30 (2010), no. 2, 399-440.

[8] K. J. Falconer, Techniques in Fractal Geometry, John Wiley and Sons, 1997.

[9] W. Li, An equivalent definition of packing dimension and its application, Nonlinear Anal. Real World Appl. 10 (2009), no. 3, 1618-1626.

[10] M. Morán, Multifractal components of multiplicative set functions, Math. Nachr. 229 (2001), 129-160.

[11] L. Olsen, A multifractal formalism, Adv. Math. 116 (1995), no. 1, 82-196.

[12] L. Olsen and S. Winter, Normal and non-normal points of self-similar sets and divergence points of a self-similar measures, J. London Math. Soc. 67 (2003), no. 3, 103-122.

[13] A. Schief, Separation properties for self-similar sets, Proc. Amer. Math. Soc. 122 (1994), no. $1,111-115$.

Department of Mathematics

Pusan University of Foreign Studies

Pusan 608-738, Korea

E-mail address: isbaek@pufs.ac.kr 